\title{
Plasma Equilibrium in Presence of the Poloidal Flow in Tokamaks
}

\author{
Ahmad Salar Elahi*, Mahmood Ghoranneviss \\ Plasma Physics Research Center, Science and Research Branch, Islamic Azad University, Tehran, Iran
}

\begin{abstract}
Main purposes of this contribution are analysis of dynamic equilibrium properties of a large aspect ratio and low beta tokamaks, in particular deriving a modified relation for the Shafranov shift in the presence of poloidal flow, and demonstrate it experimentally. Poloidal flow can produce modifications in the equilibrium properties. By increasing Alfvenic Mach number from zero, flow produce outward force, and plasma shifted in outward direction. If the poloidal Alfvenic Mach number equal to one, singularity will observe in the solution of generalized Grad-Shafranov equation. Also inversion of Shafranov shift in the transition of flow speed between sub-Alfvenic to super-Alfvenic speeds can be observed due to inward force produced by flow.
\end{abstract}

Keywords Tokamak, Equilibrium, Poloidal Flow, Shafranov Shift

\section{Introduction}

There has been an increasing attractive in the investigations of the equilibrium properties of tokamak plasmas with poloidal and toroidal flow. A precision investigation of the magnetic equilibrium is essential to interpret information from most other diagnostics and for further investigations such as Magnetohydrodynamics equilibrium and stability analysis, plasma confinement and transport studies. Also determination of the plasma equilibrium position during confinement time is essential to transport it to the control system based on feedback. However, in solving of the equilibrium problem, usually simplifying approximations have been used, e.g. axisymmetric in toroidal direction or assumption of static plasma (zero toroidal or poloidal flow), but in some cases may be these approximations not correct perfectly, for example in cases of auxiliary heating e.g. neutron beam injection, error percent can be increased with neglecting the toroidal flow. But in tokamaks which have not auxiliary heating such as the IR-T1 tokamak (see Table 1), neglecting the toroidal flow is good approximation. Instead, we suppose that poloidal flow is present in our discussion. Although this problem already had discussed for high beta tokamaks in the few works, we modified it for low beta regimes in order to use in IR-T1[1-14]. In section 2 we will be showed analytically that in presence of poloidal flows for the large aspect ratio and low beta tokamaks, changes in equilibrium properties can be observed. In the section 3 we

* Corresponding author:

salari_phy@yahoo.com (A. Salar Elahi)

Published online at http://journal.sapub.org/jnpp

Copyright (C) 2012 Scientific \& Academic Publishing. All Rights Reserved will be derived the Shafranov shift by regarding of poloidal flow. We also will be presented experimentally in section 4 , brief review for determination of the plasma position with and without regarding of the poloidal flow and also plasma rotation. Summary and conclusion are will be presented in section 5 .

\section{Tokamak Plasma Equilibrium with Poloidal Flow}

An analytic model can be derived for flowing low beta plasmas, using an expansion in terms of inverse aspect ratio $\varepsilon$. The plasma is described using the ideal isotropic magnetohydrodynamics model. The main concepts of this expansion used in here derived in[1], which will be modified to include the effect of poloidal flow in low beta tokamaks. The basic assumptions are large aspect ratio $(\varepsilon\langle<1)$, low $\beta$ ( $\beta \approx \varepsilon^{2}$ ), circular cross section, and Alfvenic poloidal flow with constant poloidal Alfvenic Mach number. For these assumptions, in addition to the usual assumption of axisymmetry we allow to write the Grad-Shafranov equation in the following generalized form[2]:

$$
\left(\mathrm{M}_{\mathrm{Ap}}^{2}-1\right) \Delta^{*} \psi=\mu_{0} \mathrm{R}^{2} \frac{\mathrm{dP}}{\mathrm{d} \psi}+\mathrm{F} \frac{\mathrm{dF}}{\mathrm{d} \psi},
$$

where $M_{A p}$ is the poloidal Alfvenic Mach number which defined below. The set of reduced equations for ideal MHD equilibria in deriving Eq. (1), the plasma flow and $\Delta^{*} \psi$ defined by:

$$
\begin{aligned}
& \vec{\nabla} .(n \vec{v})=0, \quad \vec{\nabla} \times \vec{E}=0, \quad \vec{\nabla} \times \vec{B}=\mu_{0} \vec{j}, \\
& \vec{E}+\vec{v} \times \vec{B}=0, \quad m n \vec{v} . \nabla \vec{v}=\vec{j} \times \vec{B}-\vec{\nabla} p,
\end{aligned}
$$




$$
\begin{aligned}
& \text { where } \vec{v}=M_{A \mathrm{p}} \vec{v}_{A}+R \Omega(\psi) \hat{e}_{\varphi}, \\
& M_{A \mathrm{p}}=\Phi(\psi) / \sqrt{\rho}, \\
& \Delta^{*} \psi \equiv R^{2} \vec{\nabla} \cdot\left(\frac{\vec{\nabla} \psi}{R^{2}}\right)=R \frac{\partial}{\partial R}\left(\frac{1}{R} \frac{\partial \psi}{\partial R}\right)+\frac{\partial^{2} \psi}{\partial Z^{2}}, \\
& B_{\phi}=\frac{F(\psi)}{R} \text {, and } \mathrm{B}_{\mathrm{p}}=\frac{\vec{\nabla} \psi \times \hat{e}_{\phi}}{R} .
\end{aligned}
$$

Where we used the cylindrical coordinates $(\mathrm{R}, \varphi, \mathrm{Z})$, and $\varphi(\psi), \Omega(\psi)$ are two free functions of $\psi$ describing the parallel and toroidal components of the velocity respectively. For simplicity assume that the calculation has been carried out in the toroidal coordinates $(\mathrm{r}, \theta, \varphi)$, and we have:

$$
\begin{aligned}
& \Delta^{*} \psi=\nabla^{2} \psi-\frac{\cos \theta}{\mathrm{R}} \frac{\partial \psi}{\partial \mathrm{r}}+\frac{\sin \theta}{\mathrm{rR}} \frac{\partial \psi}{\partial \theta}, \\
& \text { where } \\
& \mathrm{R}=\mathrm{R}_{0}+\mathrm{r} \cos \theta, \mathrm{Z}=\mathrm{r} \sin \theta .
\end{aligned}
$$

In the ohmically heated tokamaks (low beta regime) the assumptions in order $\varepsilon$ is given by:

$$
\begin{aligned}
& \frac{B_{p}}{B_{\varphi}} \approx \varepsilon, \quad \beta_{\mathrm{t}} \approx \frac{2 \mu_{0} p}{B_{\varphi}^{2}} \approx \varepsilon^{2}, \\
& \beta_{\mathrm{p}} \approx \frac{2 \mu_{0} p}{B_{p}^{2}} \approx 1, \quad \mathrm{q} \approx \frac{\mathrm{rB}_{\varphi}}{\mathrm{RB}_{\mathrm{p}}} \approx 1 .
\end{aligned}
$$

We can solve the generalized Grad-Shafranov equation for ohmically heated tokamaks by expanding as follows:

$$
\begin{aligned}
& \psi(\mathrm{r}, \theta)=\psi_{0}(\mathrm{r})+\psi_{1}(\mathrm{r}, \theta)+\ldots, \\
& \mathrm{p}(\psi)=\mathrm{p}_{2}\left(\psi_{0}\right)+\ldots, \\
& \mathrm{F}^{2}(\psi)=\mathrm{R}_{0}^{2} \mathrm{~B}_{0}^{2}\left[1-\frac{2 \mu_{0} \mathrm{p}_{2}(\psi)}{\mathrm{B}_{0}^{2}}+\frac{3 \mathrm{~B}_{\varphi 2}(\psi)}{\mathrm{B}_{0}}\right],
\end{aligned}
$$

where the subscripts 1 , and 2 indicate the ordering in $\varepsilon, \psi_{1}$ is the first order solution of the general GSE equation, $\mathrm{R}_{0}$ is the major radius of the plasma, $\mathrm{B}_{0}=$ const is the vacuum toroidal field at $\mathrm{R}=\mathrm{R}_{0}$, and $\mathrm{B}_{\varphi 2}(\psi)$ is a new free function replacing $\mathrm{F}(\psi)$. As shown in Eq. (5), the expressions for $\mathrm{p}(\psi)$ and $\mathrm{F}(\psi)$ are in order $\varepsilon^{2}$ (accurate for low beta regime tokamaks), as:

$$
2 \mu_{0} \mathrm{p}_{2} / \mathrm{B}_{0}^{2} \approx \varepsilon^{2}, \quad \mathrm{~B}_{\varphi 2} / \mathrm{B}_{0} \approx \varepsilon^{2} .
$$

Substituting the Eq. (3) and Eq. (5) in the Eq. (1), we can write:

$$
\begin{aligned}
\left(1-\mathrm{M}_{\mathrm{A} \mathrm{p}}^{2}\right) \nabla^{2} \psi_{1} & = \\
- & \mathrm{R}_{0}^{2} \mathrm{~B}_{0}^{2}\left[\frac{3 \mathrm{~B}_{\varphi 2}^{\prime}}{2 \mathrm{~B}_{0}}+\frac{2 \mu_{0} \mathrm{p}_{2}^{\prime} \mathrm{r}}{\mathrm{B}_{0}^{2} \mathrm{R}_{0}} \cos \theta\right],
\end{aligned}
$$

where prime denotes differentiation with respect to $\psi_{1}$.

Since Eq. (7) is a nonlinear two-dimensional partial differential equation, in approximation of radial pressure balance and toroidal force balance, for special choices of $\mathrm{B}_{\varphi 2}\left(\psi_{1}\right)$ and $\mathrm{p}_{2}\left(\psi_{1}\right)$, it can be solved analytically. A simple model is discussed below which first investigated in[3].

Consider the case where $\mathrm{B}_{\varphi 2}\left(\psi_{1}\right)$ and $\mathrm{p}_{2}\left(\psi_{1}\right)$ are linear in $\psi_{1}$ so that:

$$
\begin{gathered}
-\frac{3}{2} \mathrm{R}_{0}^{2} \mathrm{~B}_{0} \mathrm{~B}_{\varphi 2}^{\prime}\left(\psi_{1}\right)=\alpha, \\
-2 \mu_{0} \mathrm{R}_{0} \mathrm{p}_{2}^{\prime}\left(\psi_{1}\right)=\gamma,
\end{gathered}
$$

and assume the plasma surface is circular with radius a. The appropriate boundary condition is a constant value for $\psi_{1}$, which is convenient to set to zero (equal to zero pressure). The linear dependence of $\mathrm{B}_{\varphi 2}$ implies that the toroidal current density is nearly uniform. Therefore Eq. (7) reduces to:

$$
\left(1-\mathrm{M}_{\mathrm{A} \mathrm{p}}^{2}\right) \nabla^{2} \psi_{1}(\mathrm{r}, \theta)=\alpha+\gamma \mathrm{r} \cos \theta .
$$

The solution for $\psi_{1}(\mathrm{r}, \theta)$ are easily found:

$$
\psi_{1}(\mathrm{r}, \theta)=\frac{\alpha}{4 \mu}\left(\mathrm{r}^{2}-\mathrm{a}^{2}\right)+\frac{\gamma}{8 \mu}\left(\mathrm{r}^{3}-\mathrm{a}^{2} \mathrm{r}\right) \cos \theta
$$

where $\mu=1-\mathrm{M}_{\mathrm{Ap}}^{2}$.

We express the constants $\alpha, \gamma$ in terms of the physical parameters, $\mathrm{q}_{*}$ and $\beta_{\mathrm{t}}$, the results are:

$$
\begin{aligned}
& q_{*}=\frac{2 \pi \mathrm{a}^{2} B_{0}}{\mu_{0} I_{p} R_{0}}=\frac{2 \pi \mathrm{a}^{2} B_{0}}{R_{0} \int_{0}^{2 \pi} \mathrm{B}_{\theta}(a, \theta) \mathrm{ad} \theta}=\frac{3 \mu \mathrm{B}_{0}}{\alpha}, \\
& \beta_{\mathrm{t}}=\frac{2 \mu_{0}\langle\mathrm{p}\rangle}{\mathrm{B}_{0}^{2}}=\frac{2 \mu_{0}}{\pi \mathrm{a}^{2} \mathrm{~B}_{0}^{2}} \int_{0}^{2 \pi} \int_{0}^{\mathrm{a}} \mathrm{prdr} \mathrm{d} \theta= \\
& \frac{3 \mathrm{a} \gamma}{8 \mathrm{~B}_{0} \mathrm{q}_{*}}, \text { or } \alpha=\frac{3 \mu \mathrm{B}_{0}}{\mathrm{q}_{*}}, \text { and } \gamma=\frac{8 \mathrm{q}_{*} \mathrm{~B}_{0} \beta_{\mathrm{t}}}{3 \mathrm{a}} .
\end{aligned}
$$

Substituting these values in Eq. (10) leads to the following equilibrium solution of Eq. (9) for a plasma with poloidal flow, for the low beta tokamaks:

$$
\psi_{1}(r, \theta)=\xi\left[\left(\rho^{2}-1\right)+\frac{\eta}{\mu}\left(\rho^{3}-\rho\right) \cos \theta\right],
$$

where

$$
\varsigma=\frac{3 \mathrm{~B}_{0} a^{2}}{4 q_{*}}, \eta=\frac{32 q_{*}^{2} \beta_{\mathrm{t}}}{9}, \varepsilon=\frac{\mathrm{a}}{\mathrm{R}_{0}}, \rho=\frac{\mathrm{r}}{\mathrm{a}} .
$$

Where $I_{p}$ is the plasma current, $\beta_{t}$ the toroidal Beta, $q_{*}$ represents an integrated safety factor, $\eta$ is basically measure the plasma Beta, $\mu$ is measure the flow, and $\rho$ is implying a normalized minor radius.

If there are external coils to produce vertical magnetic field, the boundary condition on the flux function is modified so that we have[1]:

$$
\psi_{1}(b, \theta)=\text { const }+\psi_{1 v}(a, \theta),
$$

where $\psi_{1 v}(r, \theta)=R_{0} B_{v} r \cos \theta$ is the flux function due to external vertical field coils.

Therefore the full correction to $\psi_{1}(r, \theta)$ be:

$$
\begin{gathered}
\psi_{1 t}(r, \theta)=\psi_{1}(r, \theta)+\left(\frac{a R_{0} B_{v}}{B_{\theta 1}(a)}\right) \mathrm{B}_{\theta 1}(r), \\
B_{\theta 1}(r)=\frac{1}{R_{0}} \frac{d \psi_{0}}{d r}
\end{gathered}
$$

and $B_{v}$ is the average vertical magnetic field over the vacuum chamber. It must be noted that the solution in equation (15) is valid for both $\mu>0$, and $\mu\langle 0$. The condition $M_{A p}=1 \quad(\mu=0)$, represents however a singular point of Eq. (9), where Eq. (12) do not hold. The solution in Eq. (15), contain all the informations required to describe the equilib- 
rium, in particular the poloidal component of the magnetic field and the Shafranov shift.

\section{The Shafranov shift in Presence of the Poloidal Flow}

In this section we will present the analytical representation for the shift of the magnetic axis from the geometrical axis (the Shafranov shift). The Shafranov shift is determined by the position of the maximum of $\psi$, or in other words zero value for poloidal field inside the plasma. This can be found by solving the equation $\vec{\nabla} \psi=0$. The $\theta$ derivative can only vanish on the midplane, and this leaves again a single equation for the radial component of the gradient, which is proportional to the poloidal component of the magnetic field. The Shafranov shift can therefore be determined by solving:

$$
\vec{B}_{p}=\frac{1}{R} \vec{\nabla} \psi \times \hat{e}_{\varphi} \stackrel{\text { on magnetic axis }}{\longrightarrow} B_{p, \theta=o, \pi}=0
$$

The value of the Shafranov shift in present of the poloidal flow and external vertical field indicating with $\Delta R$, and Eq. (17) can be solved to give:

$$
\Delta R_{t}=\Delta R-\Delta R_{v}=\Delta R-\frac{a B_{v}}{B_{\theta 1}(a)},
$$

where

$$
B_{\theta 1}(a)=\frac{\mu_{0} I_{p}}{2 \pi a} .
$$

Therefore we can write:

$$
\begin{gathered}
\frac{\Delta R_{t}}{a}=\frac{-1+\sqrt{1+3\left(\frac{\eta}{\mu}\right)^{2}}}{3\left(\frac{\eta}{\mu}\right)}-\frac{2 \pi a B_{v}}{\mu_{0} I_{p}} \text { for } \mu>0, \\
\frac{\Delta R_{t}}{a}=\frac{+1-\sqrt{1+3\left(\frac{\eta}{\mu}\right)^{2}}}{3\left(\frac{\eta}{\mu}\right)}-\frac{2 \pi a B_{v}}{\mu_{0} I_{p}} \text { for } \mu\langle 0 .
\end{gathered}
$$

Therefore for flow speed lower and upper than Alfvenic speed in absence of external vertical field there is inversion of Shafranov shift.

\section{Experimental Determination of the Plasma Rotation and Equilibrium Position}

IR-T1 is a low beta, large aspect ratio, and circular cross-section tokamak (see Table (1)), which has two stainless steel grounded fully poloidal limiters with radiuses of $12.5 \mathrm{~cm}$. The main diagnostics used in this work are a diamagnetic loop and a poloidally and toroidally oriented Mach probes. The experiments were performed in hydrogen. An average plasma density was in the range $0.7-1.5 \times 10^{13}$ $\mathrm{cm}^{-3}$, the toroidal magnetic field induction $\mathrm{B}_{\mathrm{T}} \approx 0.8 \mathrm{~T}$, the plasma current $I_{p}=25-30 \mathrm{kA}$. Measurements of the plasma parameters were performed using single Langmuir probe, diamagnetic loop and a poloidally and toroidally oriented Mach probes. Theoretical models have been derived to describe the Mach probes data in un-magnetized and magnetized plasmas, using fluid, particle, and kinetic models (see the Figure (1)) [15-20]. Figure (2) shows the time dependence of the ion saturation currents collected by the poloidal Mach probes, the ratio of upstream to downstream current, and the resulting Mach number. The Mach number and thus the poloidal plasma velocity are obtained using the model of Hutchinson.
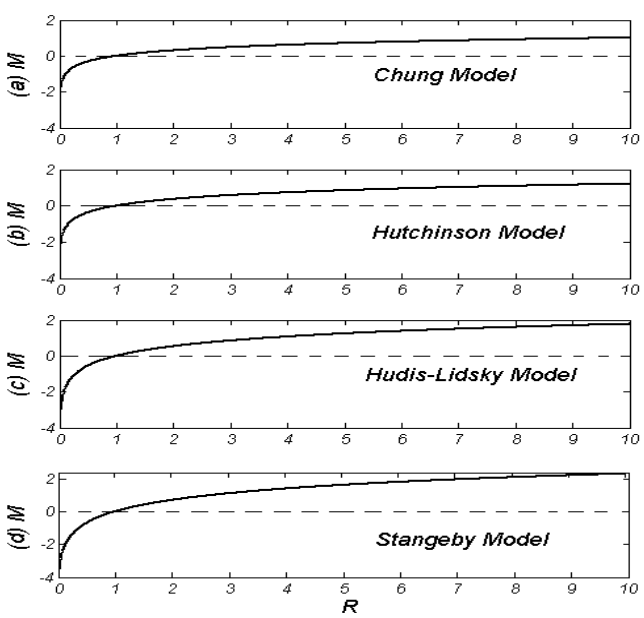

Figure 1. Theoretical models which described the ratio of ion saturation current (R) dependence of the Mach number (M), (a) the Chung model, (b) Hutchinson model, (c) Hudis-Lidsky model, and (d) the Stangeby model.

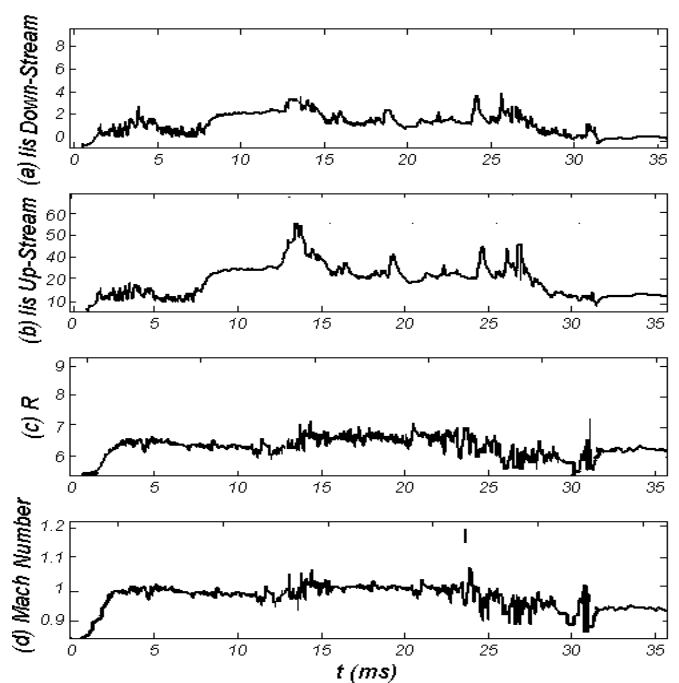

Figure 2. (a) Down-stream ion saturation current, (b) Up-stream ion saturation current, (c) Ratio of ion saturation currents, and (d) Poloidal Mach number.

For measurement of the plasma column displacement we need to obtain a toroidal beta, plasma current, vacuum toroidal field, and, moreover a vertical field. Plasma current can be measured by a Rogowski coil, vacuum toroidal field can be measured by a diamagnetic loop, and also vertical field can be measured by a saddle coil or a magnetic probe. But for determination of the toroidal Beta, from approximation for 
the ohmically heated tokamaks, we have:

$$
\beta_{t}=\varepsilon^{2} \beta_{p} / q_{*}^{2} \text {. }
$$

There are two methods for determination of the poloidal Beta: diamagnetic loop method, and magnetic probe method. We used the first method. The diamagnetic loop consists of a simple loop that links the plasma column, ideally located in a poloidal direction in order to minimize detecting the poloidal field. In cases of the ohmically heated tokamaks (low beta) where the plasma energy density is small compared to the energy density of the magnetic field, the change in the total toroidal magnetic flux is small. Therefore a reference signal equal to the vacuum toroidal magnetic flux is usually subtracted from it, giving a net toroidal flux equal to the diamagnetic flux $\Delta \Phi_{D}$ produced by the circular plasma. Relation between the diamagnetic flux and the poloidal beta derived from simplified equilibrium relation [4]:

$$
\beta_{p}=1-\frac{8 \pi \mathrm{B}_{\varphi 0}}{\mu_{0}^{2} I_{p}^{2}} \Delta \Phi_{D}
$$

where $\Phi_{\text {vacuum }}=\Phi_{T}+\Phi_{O}+\Phi_{V}+\Phi_{E}$,

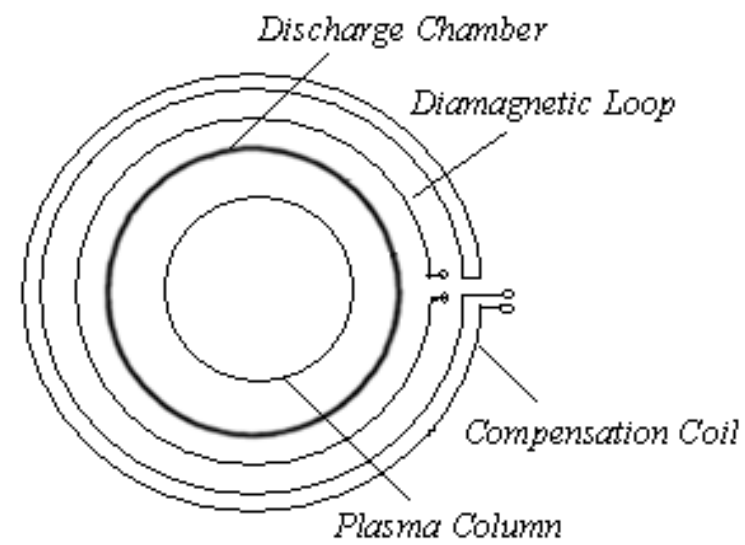

Figure 3. Positions of the diamagnetic loop with its compensation coil on outer surface of the IR-T1 Tokamak Chamber

and where $B_{\varphi 0}$ is the toroidal magnetic field in the absence of the plasma which can be obtained by the magnetic probe, $I_{p}$ is the plasma current which can be obtained by the rogowski coil, $\Phi_{T}$ is the toroidal flux because of toroidal field coils, $\Phi_{O}$ and $\Phi_{V}$ are the passing flux through loop due to possible misalignment between ohmic field and vertical field and the diamagnetic loop and $\Phi_{E}$ is the toroidal field due to eddy current on the vacuum chamber. These fluxes can be compensated either with compensation coil or fields discharge without plasma. It must be noted that compensating coil for diamagnetic loop is wrapped out of the plasma current, and only the toroidal flux (which is induced by the change of toroidal field coil current when plasma discharges) can be received (see Figure (3)). As shown in Eq. (23) the diamagnetic loop signal contains two parts, plasma diamagnetic flux and the vacuum toroidal flux. So the diamagnetic flux $\Delta \Phi_{D}$ caused by plasma current can be measured from the diamagnetic and compensating coil using subtraction.
Table 1. Main Parameters of the IR-T1 Tokamak

\begin{tabular}{|c|c|}
\hline Parameters & Value \\
\hline Major Radius & $45 \mathrm{~cm}$ \\
\hline Minor Radius & $12.5 \mathrm{~cm}$ \\
\hline Toroidal Field & $\langle 1.0 \mathrm{~T}$ \\
\hline Plasma Current & $\langle 40 \mathrm{kA}$ \\
\hline Discharge Time & $\langle 35 \mathrm{~ms}$ \\
\hline Electron Density & $0.7-1.5 \times 10^{13} \mathrm{~cm}^{-3}$ \\
\hline
\end{tabular}

By substituting the toroidal beta obtained from diamagnetic loop, plasma current measured using the Rogowski coil, vacuum toroidal field obtained from diamagnetic loop, the average vertical field obtained from Saddle coil, and different values of Alfvenic Mach number in Eqs. (15), (18), and (19), magnetic flux surfaces and displacement of plasma column center obtained as shown in Figures (4), (5), (6), and (7).

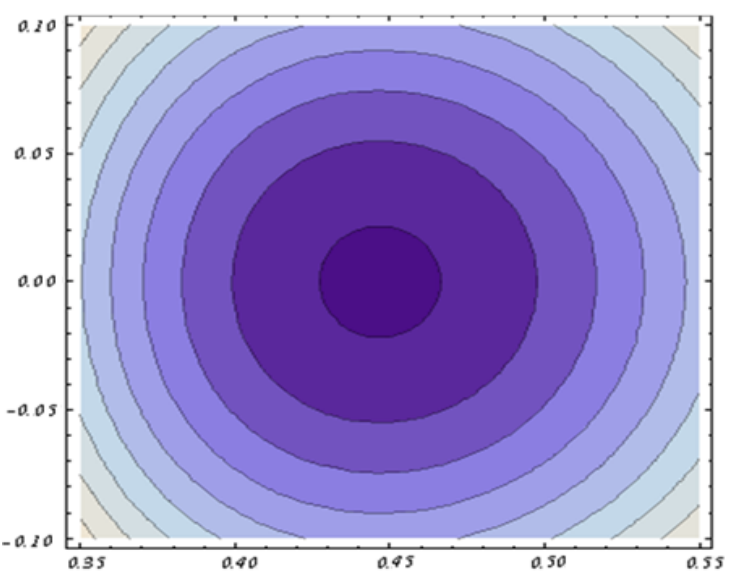

Figure 4. Magnetic Flux Surfaces for $\mathrm{t}=15 \mathrm{~ms}$ in target shot in IR-T1 tokamak, in case of Alfvenic Mach number $\left(M_{A P}\right)$ equal to zero.
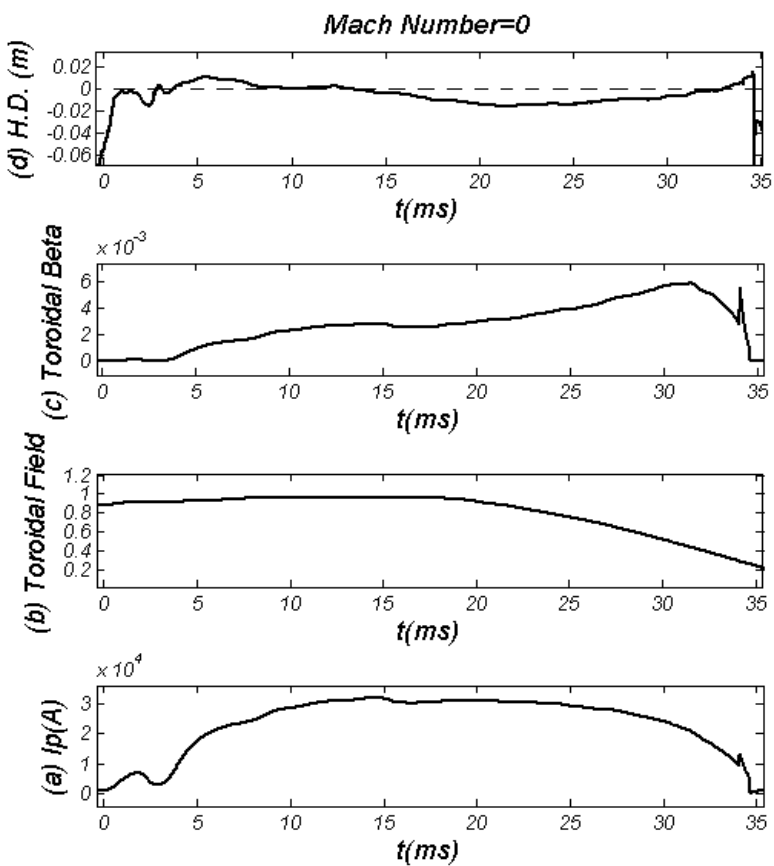

Figure 5. (a) Plasma Current, (b) Toroidal Magnetic Field, (c) Toroidal Beta, and (d) Horizontal Displacement (H.D.) in case of that Poloidal Alfvenic Mach number $\left(\mathrm{M}_{\mathrm{AP}}\right)$ equal to zero in IR-T1 tokamak 

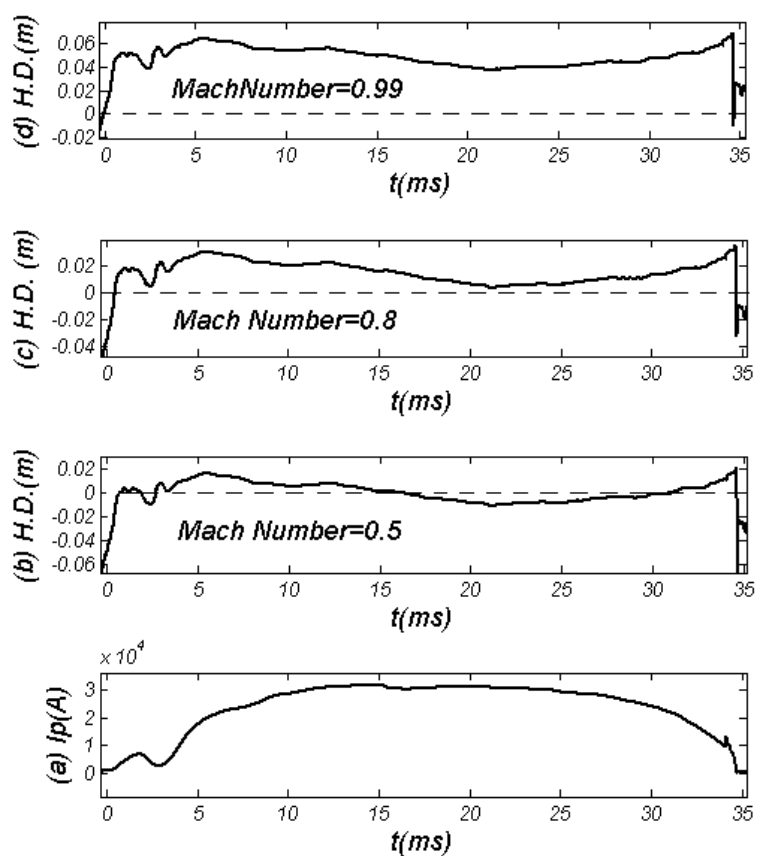

Figure 6. Effects of Alfvenic Mach number $\left(\mathrm{M}_{\mathrm{AP}}\right)$ on Horizontal Displacement (H.D) in IR-T1 Tokamak, (a) Plasma Current, (b) H.D in case of that $\mathrm{M}_{\mathrm{AP}}=0.5$ (c) H.D in case of $\mathrm{M}_{\mathrm{AP}}=0.8$, and (d) H.D in case of $\mathrm{M}_{\mathrm{AP}}$ $=0.99$. Increasing the values of the $\mathrm{M}_{\mathrm{AP}}$, flow produce outward force (plasma column displacement is increased in outward direction)
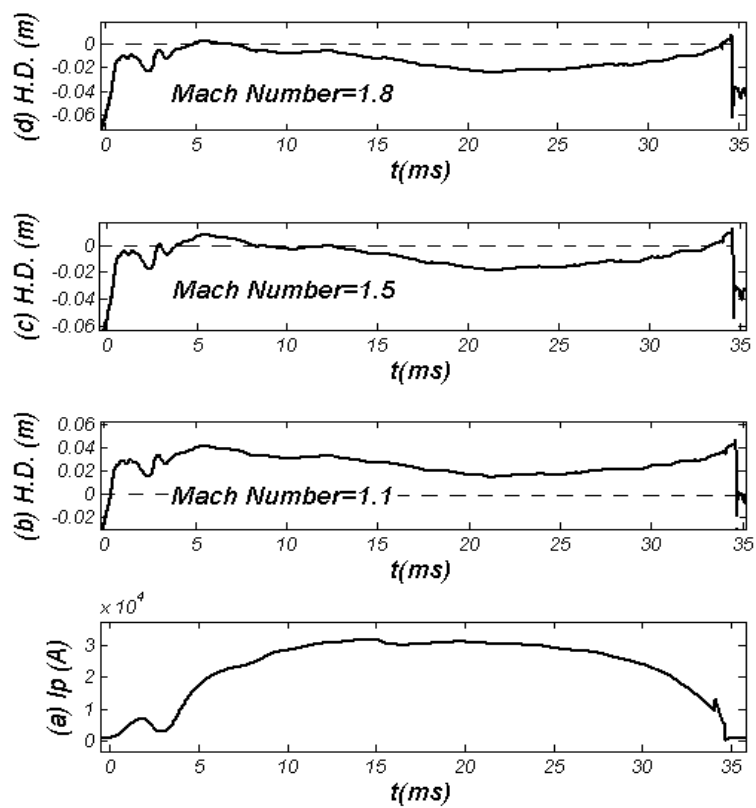

Figure 7. Effects of Alfvenic Mach Number (AMn) on Horizontal Displacement (H.D) in IR-T1 Tokamak, (a) Plasma Current, (b) H.D in case of that $\mathrm{M}_{\mathrm{AP}}=1.1$ (c) H.D in case of $\mathrm{M}_{\mathrm{AP}}=1.5$, and (d) H.D in case of $\mathrm{M}_{\mathrm{AP}}=1.8$. Increasing the values of $\mathrm{M}_{\mathrm{AP}}$ from 1, flow produce inward force (plasma column displacement is inverted).

\section{Summary and Conclusions}

In this paper we presented analysis of dynamic equilibrium properties of tokamaks, in particular deriving a modi- fied relation for the Shafranov shift in the presence of Alfvenic poloidal flow and external vertical field, for large aspect ratio and low beta tokamaks. Results demonstrated experimentally on the IR-T1, which is the large aspect ratio and ohmically heated tokamak. In the presence of Alfvenic poloidal flows, singularity in equilibrium observed. By increasing the value of Alfvenic Mach number from zero, flow produces outward force. In the transition of flow speed between lower to upper than the Alfven speed, the Shafranov shift can be inverted due to inward force produced by flow.

\section{REFERENCES}

[1] J. P. Freidberg, Ideal MHD (Clarendon, Oxford, 1987)

[2] L. Guazzotto and R. Betti, Phys. Plasmas 12, 056107 (2005)

[3] J. P. Freidberg and F. A. Haas, Phys. Fluids 16, 1909 (1973)

[4] I. H. Hutchinson, Principles of Plasma Diagnostics, (Cambridge University Press, Cambridge, 1987)

[5] L. Zakharov, Am. Bulletin, Phys. Society, Division of Plasma Physics, 45, 225 (2000)

[6] W. Zhang, Z. Wu and D. Li, Phys. Plasmas 12, 042106 (2005)

[7] H. Tasso and G. N. Throumoulopoulos, Phys. Plasmas 5, 2378 (1998)

[8] J. P. Graves, R. J. Hastie and K. I. Hopcraft, Plasma Phys. Control. Fusion 42, 1049 (2000)

[9] J. E. Rice, W. D. Lee, E. S. Marmar et al., Phys. Plasmas 11, 2427 (2004)

[10] E. J. Strait and et al., Fusion Science and Technology, 53, pp. 304-330, (2008)

[11] A. Salar Elahi and et al., 2009, J Fusion Energy 28 (4), DOI 10.1007/s10894-009-9198-X.

[12] A. Salar Elahi and et al., Phys. Scripta 80, 045501 (2009), DOI 10.1088/0031-8949/80/04/045501

[13] A. Salar Elahi and et al., Phys. Scripta 80, 055502 (2009), DOI 10.1088/0031-8949/80/05/055502

[14] A. Salar Elahi and et al., IEEE T on Plasma Sci. 38 (2), DOI 10.1109/TPS.2009.2037965

[15] I. H. Hutchinson, Phys. Fluids 30, 3777 (1987).

[16] I. H. Hutchinson, Phys. Rev. A 37, 4358 (1988).

[17] B. J. Peterson et al., Rev. Sci. Instrum. 65 (8), 1994, 2599

[18] P. C Stangeby, Phys. Fluids 27, 2699 (1984)

[19] K. S. Chung et al., Phys. Rev. A 38, 4721 (1988)

[20] K. S. Chung et al., Phys. Fluids B 1, 2229 (1989) 